\title{
Article \\ Quality of Service Based NOMA Group D2D Communications
}

\author{
Asim Anwar ${ }^{+}$(D), Boon-Chong Seet $*,+$ iD and Xue Jun Li ${ }^{+}$(iD \\ Department of Electrical and Electronic Engineering, Auckland University of Technology, Auckland 1010, \\ New Zealand; asim.anwar@aut.ac.nz (A.A.); xuejun.li@aut.ac.nz (X.J.L.) \\ * Correspondence: boon-chong.seet@aut.ac.nz \\ + These authors contributed equally to this work.
}

Received: 6 October 2017; Accepted: 26 October 2017; Published: 1 November 2017

\begin{abstract}
Non-orthogonal multiple access (NOMA) provides superior spectral efficiency and is considered as a promising multiple access scheme for fifth generation (5G) wireless systems. The spectrum efficiency can be further enhanced by enabling device-to-device (D2D) communications. In this work, we propose quality of service (QoS) based NOMA (Q-NOMA) group D2D communications in which the D2D receivers (DRs) are ordered according to their QoS requirements. We discuss two possible implementations of proposed Q-NOMA group D2D communications based on the two power allocation coefficient policies. In order to capture the key aspects of D2D communications, which are device clustering and spatial separation, we model the locations of D2D transmitters (DTs) by Gauss-Poisson process (GPP). The DRs are then considered to be clustered around DTs. Multiple DTs can exist in proximity of each other. In order to characterize the performance, we derive the Laplace transform of the interference at the probe D2D receiver and obtain a closed-form expression of its outage probability using stochastic geometry tools. The performance of proposed Q-NOMA group D2D communications is then evaluated and benchmarked against conventional paired D2D communications.
\end{abstract}

Keywords: quality of service; device-to-device communication; non-orthogonal multiple access; stochastic geometry

\section{Introduction}

With the advancement in mobile communication research, the usage of cellular technology has spread beyond voice and simple data transfer to high data rate, delay sensitive, and loss tolerant multimedia applications. Despite the fast growth of fourth generation (4G) systems, the current spectrum resources are still scarce to meet the ever increasing subscribers' demands for bandwidth and resource hungry applications, with vigorous requirements of seamless connectivity, anywhere and anytime. These trends compelled wireless researchers from the academia and industry to define new paradigm technologies and structures to achieve the goals of fifth generation (5G) systems [1,2].

In order to realize the concept of $5 \mathrm{G}$ into reality, many enabling technologies are proposed, among which millimetre waves, massive multiple-input multiple-output (MIMO), full-duplex (FD), heterogeneous deployments and software-defined networks have captured the attention of both academia and industry [2,3]. Nevertheless, the role of multiple access scheme always remains a vital factor in cellular networks in order to enhance the system capacity in a cost effective manner, while utilizing the bandwidth in such a way that overall spectral efficiency will be increased [4].

Non-orthogonal multiple access (NOMA) is considered as a promising multiple access candidate for future fifth generation (5G) wireless systems due to its potential of improving spectrum efficiency $[5,6]$. Unlike conventional orthogonal multiple access (OMA), NOMA superimposes message signals of different users in power domain and send this conglomerate signal using the same time, 
frequency or code resource $[7,8]$. Successive interference cancellation (SIC) technique is employed at each receiver to cancel the intra-user interference [9].

Apart from NOMA, another emerging technique to enhance the spectral efficiency is device-to-device (D2D) communications [10], which has the ability to improve the spectral efficiency of the conventional cellular network by sharing same spectrum resources among cellular users and D2D pairs [11-13].

By introducing aforementioned two concepts and their potential to improve spectrum efficiency, it is natural to investigate the application of NOMA scheme to D2D communications.

\subsection{Related Work and Motivation}

Recently, a FD D2D aided cooperative NOMA is proposed in [14]. The base station (BS) sends a NOMA signal to one NOMA-strong and one NOMA-weak user, where the strong user is equipped with full-duplex ability. By invoking D2D communication between strong and weak NOMA user pair, the authors proposed to improve the outage performance of the weak user with the aid of D2D aided direct and cooperative transmissions. However, they only considered a single-cell scenario where NOMA is conducted at BS while strong and weak users communicate via conventional paired D2D communication. The authors in [15] developed an analytical framework based on stochastic geometry to analyse cellular networks with underlay D2D communications. The D2D users are also equipped with FD transceivers and can operate in FD mode. The authors proposed criteria to select between FD and D2D modes of operation. They derived closed-form expression for outage probability to evaluate the performance of cellular and D2D users. However, they modeled spatial topology of D2D users by a Poisson point process (PPP), which may not be realistic distribution choice for D2D users. The reason is that PPP cannot capture the features of device clustering and spatial separation of D2D communications due to its completely random nature [16]. Furthermore, NOMA protocol is not applied for both cellular and D2D communications. A relay assisted diversity communication is proposed in [17]. The proposed analytical framework analyses the frame error probability performance by considering the effects of node locations, link characteristics, power allocation, diversity methods and distributed coding and constellation signaling. However, they considered an OMA based communication between single source and destination assisted by three relay nodes. The authors in [18] considered a downlink multiuser MIMO NOMA celullar network with underlaid D2D communications. They proposed two beamforming schemes in order to eliminate the inter-beam interference and the one caused to D2D users by BS transmission. In addition, they formulated an optimization problem to jointly study the performance of both cellular and D2D users. A potential limitation to their approach is that the considered system model is limited to single cell and no specific random distribution is utilised to model the spatial topologies of cellular and D2D users. Hence, it is not straightforward to generalize the results for a case of multi-cell network. Furthermore, they considered a paired D2D communication where D2D users do not apply NOMA protocol to communicate with each other.

In [19], the authors considered a NOMA-based D2D communications and introduced the concept of D2D group, where a D2D transmitter (DT) is communicating with multiple D2D receivers (DRs) using NOMA protocol. The authors proposed an optimal resource allocation strategy for interference management that enables to realize the NOMA-based D2D group communications. Although the concept of NOMA group D2D was introduced in [19], their system model was comprised of single-cell and lack of interference characterization at DR. Furthermore, it requires interference modeling and performance evaluation at the DR in order to extend the concept of NOMA group D2D to a general scenario, where DTs and DRs are distributed in the entire network. The authors in [20] further proposed cooperative hybrid automatic repeat request (HARQ) assisted NOMA in large-scale D2D networks. They studied the outage and throughput performance of the D2D users under the considered network setting and demonstrated that cooperative HARQ assisted NOMA achieves lower outage probability than non-cooperative case and OMA scheme. However, their NOMA based D2D network model is restricted to the two-user case only i.e., they only considered two-user 
NOMA transmission from D2D sources. Furthermore, they considered a significant difference between channels of two D2D users by assuming that one user is always closer to D2D source compared to the other user. This assumption may not always hold, particularly in the scenario of NOMA based D2D communications because DRs are clustered around DTs and are located in proximity of each other and hence DRs may have very similar channel conditions.

In the light of the abovementioned discussion, very little attention was paid to investigate NOMA group D2D communications. Hence, the aforementioned gaps and shortcomings motivated us to investigate and analyse the performance of NOMA based group D2D communications under interference limited scenarios. In order to capture the key features of D2D communications, i.e., device clustering and spatial separation, the DTs are considered to be randomly distributed over $\mathbb{R}^{2}$ according to Gauss-Poisson process (GPP), while the DRs are assumed to be randomly clustered around DTs. The reason for choosing GPP is that it is a relatively simpler cluster point process that maintains good trade-off between modeling accuracy and analytical tractability. Therefore, in the context of D2D communications, GPP provides more realistic modeling approach against PPP case by capturing the clustering behavior of D2D devices [21].

Furthermore, the current approach to order users in NOMA group D2D communications is based on the channel gains of the DRs. This ordering approach may not be suitable to D2D communication scenario under which the DRs in the same group are clustered around a common DT and are located in proximity of each other. Hence, the channel conditions of the DRs located in the same D2D group would be very similar. Consequently, this ordering strategy may result in very similar power allocation, which could limit the gains of applying NOMA to D2D communications [7]. In the context of NOMA, there are few works that use quality of service (QoS) based ordering [22-24]. They mainly focused on cellular networks and are limited to the two-user case only. In this paper, we propose and analyse QoS based NOMA (Q-NOMA) group D2D communications and make an attempt to fill the aforementioned gaps in literature. To the best of our knowledge, it is the first time that Q-NOMA is proposed and analysed to realize group D2D communications under interference limited scenario.

\subsection{Contributions}

The main contributions of this work are as follows:

- We propose Q-NOMA group D2D communications in which D2D users are randomly distributed over the entire two-dimensional plane. Unlike the existing proposals, we order the DRs according to their QoS requirements, which is more appropriate for the D2D communications scenario. Furthermore, in contrast to PPP, which is most commonly used to model D2D users (both DTs and DRs), we model the spatial topology of DTs by GPP and DRs are considered to be randomly clustered around DTs. These spatial distributions of DTs and DRs are suitable to analyse the proposed network with any number of D2D users. In addition, based on the QoS ordering, we propose two policies to compute power allocation coefficients that could lead to two implementations of the proposed Q-NOMA group D2D communications.

- We derive the interference distribution at the probe DR by utilizing the results from stochastic geometry. The Laplace transform of interference over GPP is derived in [21], which involves complex double integrals. In order to obtain useful insights, a major step in characterizing the interference is the approximation of integrals in the interference Laplace transform by applying Gaussian-Chebyshev and Gauss-Laguerre quadratures. This approximation results in an interference Laplace transform expression, which is easy to implement.

- Based on the interference approximation results, we further derive the closed-form expression for outage probability of the DRs in the proposed Q-NOMA group D2D communications.

- We present numerical results to validate the accuracy of the derived outage results and compare the performance of the proposed Q-NOMA group D2D with conventional paired D2D communications using OMA. 


\subsection{Mathematical Preliminaries on Gauss-Poisson Process}

The generalized GPP [21] is defined as the Poisson cluster process with homogeneous independent clustering. Denote $\lambda$ as the parent process intensity. Then, each cluster in GPP can be classified as single-point or two-point cluster. Let $1-a$ and $a$ denote the probabilities that a cluster is single-point, and two-point, respectively. When the cluster is single-point, the point is located at the position of the parent. When the cluster is two-point, one point is located at the position of the parent while the other is randomly distributed around the parent with some probability density function (PDF) $f_{u}(\cdot)$, where $u$ is the inter-point distance in two-point cluster.

\section{System Model}

Consider inband D2D communications with an overlay cellular network. We consider a frequency reuse factor of one among D2D users to achieve better spectrum efficiency. With this setting, every D2D transmission by a DT is subjected to interference from other active DTs. We consider a composite fading and path loss channel model between every DT and DR. In this work, we assume that the power fading coefficients are independent and identically distributed (i.i.d) with exponential distribution of unit mean, and adopt a path loss model of $d^{-\alpha}$, where $d$ is the distance between the probe DR and test DT, and $\alpha$ is the path loss exponent.

\subsection{Spatial Distribution of D2D Users}

Consider that D2D users are randomly distributed over $\mathbb{R}^{2}$. At any time realization, the D2D users are classified as transmitters or receivers. We consider the group D2D scenario, where each DT is communicating with multiple DRs via a NOMA scheme. We refer to a DT communicating to multiple D2D devices as a group transmitter (GT). Any D2D user can take a role of GT. We assume that the selection of GTs is performed by a BS, and it can select multiple GTs in a given cell to improve overall system capacity. We allow multiple GTs to exist in proximity of each other, where each GT is communicating to its own group of receivers. Hence, at any time realization, each selected GT forms a group/cluster containing DRs. In order to capture both inband and device clustering, we model the spatial topology of the GTs by a stationary and isotropic GPP defined on $\mathbb{R}^{2}$, denoted by $\Phi_{\mathrm{GT}}$ with parent process intensity $\lambda_{\mathrm{GT}}$. Furthermore, we model the coverage of each GT by a disc $D$ with radius $R_{D}$. We consider that the DRs are clustered around each GT and are uniformly distributed inside coverage of GTs. An illustration of NOMA group D2D communication is presented in Figure 1. For a quick reference, a list of commonly used abbreviations is given in Table 1 .

Table 1. List of commonly used abbreviations.

\begin{tabular}{ll}
\hline D2D & Device-to-device \\
DR & D2D receiver \\
DT & D2D transmitter \\
F-NOMA & Fixed NOMA \\
GPP & Gauss-Poisson process \\
GT & Group transmitter \\
NOMA & Non-orthogonal multiple access \\
PPP & Poisson point process \\
QoS & Quality of service \\
SIC & Successive interference cancellation \\
SNR & Signal to noise ratio \\
\hline
\end{tabular}




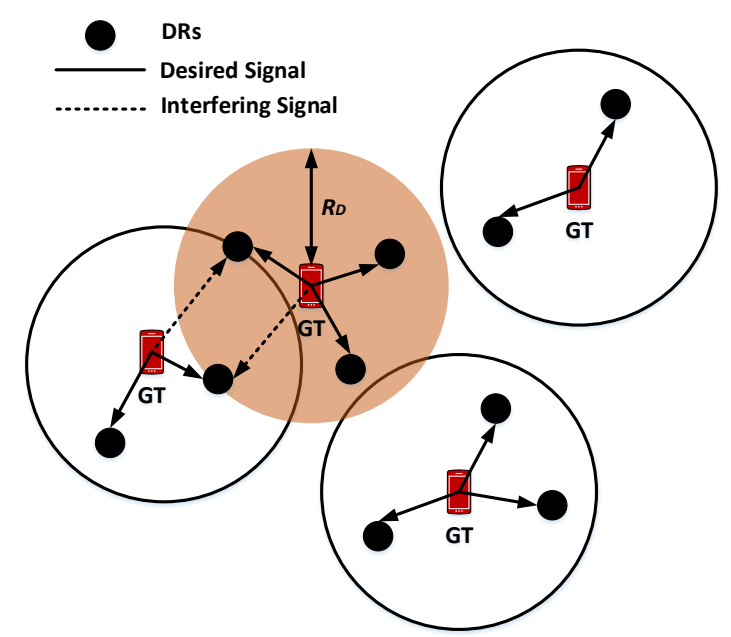

Figure 1. Example of inband non-orthogonal multiple access (NOMA) group device-to-device (D2D) communications with overlay cellular network.

\subsection{Q-NOMA Group D2D Communication}

Since DRs are clustered around GTs and are located in proximity of each other, therefore, the DRs connected to same GT would have very similar channel conditions. Consequently, in the context of D2D communications, ordering DRs according to their channel conditions to apply NOMA at GT may not achieve the desired multiplexing gains and fairness among DRs. Hence, in this work, we propose ordering the DRs of the GT according to QoS requirements, which are determined by their targeted data rates.

Let there be a total of $M$ DRs distributed inside coverage of a test GT. The probe DR is assumed to be located at the origin, with a desired test GT at $x_{0}=(d, 0)$ with $d \neq 0$. Without loss of generality, we assume that the DRs are ordered as $R_{1} \leq \ldots \leq R_{M}$, where $R_{i}$ is the targeted data rate of DR $i$, $1 \leq i \leq M$. Correspondingly, the power allocation coefficients are sorted as $\beta_{1} \geq \ldots \geq \beta_{M}$.

The aforementioned procedure to order DRs of the GT and compute their power allocation coefficients according to users' targeted rates is termed as "Q-NOMA". When it is applied to D2D communications, we refer to the communication as "Q-NOMA group D2D communication".

Consider that the NOMA DR $m$ is the probe receiver, then, the received signal from the test transmitter at the probe DR is given as:

$$
y_{m}=h_{m} \sum_{i=1}^{M} \sqrt{\beta_{i} P_{\mathrm{GT}}} s_{i}+n_{m}
$$

where $P_{\mathrm{GT}}$ is the transmission power of test GT, $s_{i}$ is the message signal of DR $i$ and $n_{m}$ is the additive white Gaussian noise (AWGN) with zero mean and variance $\sigma^{2}$.

\subsection{Power Allocation Coefficients Policies}

The optimal power and resource allocation improve overall performance and utilise the system resources efficiently. However, the optimum power allocation strategies proposed in existing literature, for example, [25], cannot be directly applied to the current work because of significant difference in system model or underlying transmission method. Therefore, in this sub-section, we discuss two simple methods to compute power allocation coefficients $\left\{\beta_{i}\right\}_{i=1}^{M}$ that would lead to two possible implementations of Q-NOMA group D2D communications. 


\subsubsection{Policy I}

The Policy I utilises targeted rates of DRs to compute the power allocation coefficients. Similar to [7], the power allocation coefficient for DR $i$ under Policy I is computed as:

$$
\beta_{i}=\frac{1 / R_{i}}{\sum_{j=1}^{M} \frac{1}{R_{j}}}
$$

The intuition behind Equation (2) is that power allocation coefficients could be utilised to maintain fairness among DRs $[3,26]$. In case they are computed in proportion with users' targeted rates, then the highest ordered DR $M$ would result in the highest SIC decoding order with maximum signal-to-interference-plus-noise ratio (SINR) threshold among all ordered DRs. Consequently, all of the lower ordered DRs always require the maximum SINR threshold dictated by the user $M$, which would result in a biased treatment of lower ordered users (with lower targeted rates). Hence, in order to avoid this biasness, we propose to compute the power allocation coefficients as given by Equation (2).

\subsubsection{Policy II}

The concept of fixed NOMA (F-NOMA) was proposed in [27], where the power allocation coefficients are fixed and are computed based on the given user ordering i.e., it does not utilise the actual channel gains to compute $\left\{\beta_{i}\right\}_{i=1}^{M}$. Similar to [27], we adopt a Policy II to compute $\left\{\beta_{i}\right\}_{i=1}^{M}$ for conventional NOMA that does not utilise the actual targeted rates of DRs. The power allocation coefficient for DR $i$ under Policy II is computed as:

$$
\beta_{m}^{\mathrm{F}}=\frac{M-m+1}{\mu}
$$

where $\beta_{m}^{\mathrm{F}}$ represents the power allocation coefficient of DR $m$ in Policy II and $\mu$ is selected in such a way that $\sum_{i=1}^{M} \beta_{i}^{\mathrm{F}}=1$.

\subsection{Interference Distribution}

The reception at the probe DR from the test GT is interfered by the other GTs. The interference at probe DR is given as, $I=\sum_{x \in \Phi_{\mathrm{GT}} \backslash x_{0}}\left|g_{x}\right|^{2} d_{x}^{-\alpha}$, where $g_{x}$ and $d_{x}$ represent the Rayleigh fading channel gain and distance between probe DR and interferer at $x_{0}$, respectively. The following lemma provides the Laplace transform of the interference at probe DR.

Lemma 1. Consider a GPP $\Phi_{G T}$ with parent process intensity $\lambda_{G T}$ modeling spatial topology of the GTs in a $Q-N O M A$ group D2D communications. Then, the Laplace transform of the interference at the probe DR conditioned at the location of test GT is given by:

$$
\mathcal{L}_{I}(s)=e^{-2 \pi \lambda_{G T} \sum_{p=1}^{P} \Omega_{p} \frac{a\left(1-X_{1}\left(r_{p}\right)\right)+s r_{p}^{-\alpha}}{1+s r_{p}^{-\alpha}}} \cdot \Lambda_{2}(d),
$$

where $\Omega_{p}=\omega_{p} e^{r_{p}}, \omega_{p}=\frac{\Gamma(P+2) r_{p}}{P !(P+1)^{2}\left(L_{P+1}\left(r_{p}\right)\right)^{2}}, L_{P}(\cdot)$ is the Laguerre polynomial of degree $P, r_{p}$ are the roots of $L_{P}(\cdot), X_{1}(\cdot)$ and $\Lambda_{2}(\cdot)$ are given in Equations $(A 7)$ and $(A 10)$, respectively.

Proof. See Appendix A.

\section{Outage Probability Analysis}

In this section, we focus on the outage probability for the DRs in the considered Q-NOMA group D2D communication. Let $\tau_{m}$ and $R_{m}$ denote the SINR threshold and targeted rate of DR $m$, respectively, 
where $\tau_{m}=2^{R_{m}}-1$. Since each DR employs SIC, the outage at DR $m$ occurs if it does not meet the targeted rate of any higher order DR $j$, where $1 \leq j<m$. Denote $\zeta_{m \rightarrow j}=\left\{\tilde{R}_{m, j}<R_{j}\right\}$ as the outage event at DR $m$ due to decoding of DR $j$, where $\tilde{R}_{m, j}$ is the achievable rate of user $j$ at DR $m$. The outage event $\zeta_{m \rightarrow j}$ can be expressed as:

$$
\begin{aligned}
\zeta_{m \rightarrow j} & =\left\{\tilde{R}_{m, j}<R_{j}\right\} \\
& =\left\{\log _{2}\left(1+\frac{h_{m} \beta_{j} P}{h_{m} P \sum_{i=j+1}^{M} \beta_{i}+I+\sigma^{2}}\right)<R_{j}\right\} \\
& =\left\{h_{m}<\frac{\varphi_{j}(\rho I+1)}{\rho_{t}}\right\},
\end{aligned}
$$

where $\varphi_{j}=\frac{\tau_{j}}{\beta_{j}-\tau_{j} \sum_{i=j+1}^{M} \beta_{i}}, \rho=\frac{P_{I}}{\sigma^{2}}, \rho_{t}=\frac{P_{\mathrm{GT}}}{\sigma^{2}}$ is the transmit signal-to-noise ratio (SNR) and $P_{I}$ is the maximum received interference power at the probe DR.

Next, define $\varphi_{m}^{\max }=\max \left\{\varphi_{1}, \ldots, \varphi_{m}\right\}$. Based on Equation (3), the outage probability at the DR $m$ can be given as:

$$
\begin{aligned}
\mathbb{P}_{m} & =\operatorname{Pr}\left(h_{m}<\frac{\varphi_{m}^{\max }(\rho I+1)}{\rho_{t}}\right) \\
& =\mathbb{E}_{I}\left[F_{h_{m}}\left(\frac{\varphi_{m}^{\max }(\rho x+1)}{\rho_{t}}\right)\right],
\end{aligned}
$$

where $F_{h_{m}}$ is the cumulative distribution function (CDF) of $h_{m}$.

Note that the set $\left\{h_{i}\right\}, i=1, \ldots, M$, of channel gains is not ordered because the users are sorted in ascending order of their targeted rates. Since the channel gains are i.id. random variables with common $\mathrm{CDF} F_{h}$, Equation (4) can be re-written as:

$$
\mathbb{P}_{m}=\mathbb{E}_{I}\left[F_{h}\left(\frac{\varphi_{m}^{\max }(\rho x+1)}{\rho_{t}}\right)\right]
$$

Consequently, the outage probability of DR $m$ is provided in the following theorem.

Theorem 1. The outage probability of DR $m$ in the Q-NOMA group D2D communications is derived as:

$$
\mathbb{P}_{m}=\sum_{l=1}^{L} b_{l} e^{-\frac{\varphi_{m}^{\max } \rho_{l}}{\rho_{t}}} \mathcal{L}_{I}\left(\frac{\varphi_{m}^{\max } c_{l} \rho}{\rho_{t}}\right)
$$

where $b_{l}=\omega_{l} \sqrt{1-\phi_{l}^{2}}\left(1+\phi_{l}\right), \omega_{l}=\frac{\pi}{L}, c_{l}=\left(\frac{R_{D}}{2}\left(1+\phi_{l}\right)\right)^{\alpha}, \phi_{l}=\cos \left(\frac{(2 l-1) \pi}{2 L}\right)$ and $L$ is the complexity-accuracy trade-off parameter.

Proof. See Appendix B.

Note that, due to the presence of interferers in the network, similar to [20,28], the derived outage probability in Equation (8) is a function of variables $\varphi_{m}^{\max }$ and interference Laplace transform $\mathcal{L}_{I}$. This is different from existing works that analyse NOMA wireless systems under no interference where outage probability is mainly a function of $\varphi_{m}^{\max }$. For reference, please see $[14,27,29]$. Furthermore, it is worthy to note that the current outage analysis approach remains valid if more complex cluster models (Poisson cluster process, etc.) are adopted for modeling spatial distributions of DTs and DRs. In that case, the $\mathcal{L}_{I}$ term will be replaced by the Laplace transform of the interference for the adopted model. Intuitively, $\mathcal{L}_{I}$ is performing a form of scaling in Equation (8) and hence the conclusions are expected to remain the same if the spatial distribution model(s) of the DTs and DRs is changed. However, the exact 
impact on outage probability when more complex cluster processes model is adopted for modeling DT and DR locations would require further study, and we plan to investigate them in our future work.

\section{Numerical Results and Discussion}

This section presents the numerical results to evaluate the performance of the considered network as well as to validate the accuracy of the derived expression in Equation (8) of Section 3. As shown in Table 2, simulation parameters used are similar to those in [29], unless otherwise stated. Furthermore, we adopt Policy I as a default policy to compute power allocation coefficients, unless otherwise stated.

Table 2. Simulation parameters.

\begin{tabular}{ccc}
\hline Parameter & Description & Value \\
\hline$M$ & Total users & 3 \\
$\left\{R_{m}\right\}_{m=1}^{M}$ & Users' targeted rates & $\{0.7,1.1,2\}$ \\
$R_{D}$ & Coverage of GT & $10 \mathrm{~m}$ \\
$\alpha$ & Path loss exponent & 4 \\
$\lambda_{\mathrm{GT}}$ & Intensity of GTs & $10^{-4}$ \\
$\rho_{t}$ & SNR range & $(5-40) \mathrm{dB}$ \\
$L, N, V, Q, S$ & Gaussian-Chebyshev parameters & 5 \\
$P$ & Degree of Gauss-Laguerre polynomial & 5 \\
$d$ & Distance between probe DR and GT & $5 \mathrm{~m}$ \\
\hline
\end{tabular}

\subsection{Impact of $R_{D}$ on Outage Probability}

Figure 2 presents the impact of varying coverage radius $R_{D}$ of test GT on the outage probability of ordered DRs as a function of SNR. The derived outage results in Equation (8) are shown to be in good agreement with the Monte Carlo simulations.

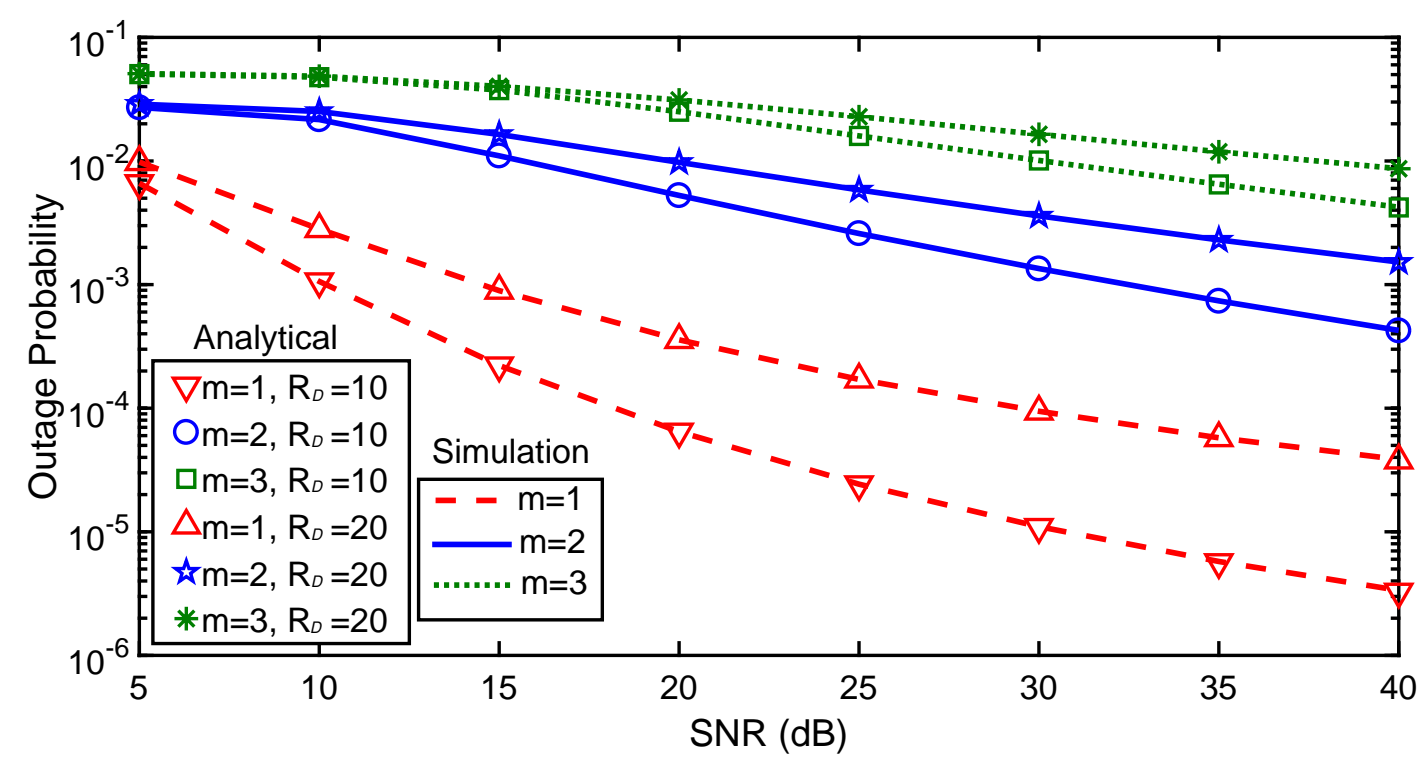

Figure 2. Impact of $R_{D}$ on outage probability.

Several observations can be made from the results in Figure 2: (1) increasing the coverage radius of GT results in a higher outage probability because of a larger path loss; (2) different ordered users have distinct decreasing slopes of outage probability because of different targeted rates; (3) the higher order DRs in our proposed Q-NOMA group D2D communications achieve better outage performance because they have smaller targeted rates. This is different from conventional NOMA that orders users 
based on channel conditions, and where the higher ordered users have larger outage probabilities due to poor channel conditions $[27,28]$.

\subsection{Impact of $d$ on Outage Probability}

The impact of varying distance between probe DR and test GT on the outage performance is investigated in Figure 3. It can be observed that varying $d$ has a larger impact on the average achievable outage probability at lower SNR value of $5 \mathrm{~dB}$. This is intuitively plausible because increasing transmission power results in improved SINR at the receiver and hence better outage performance.

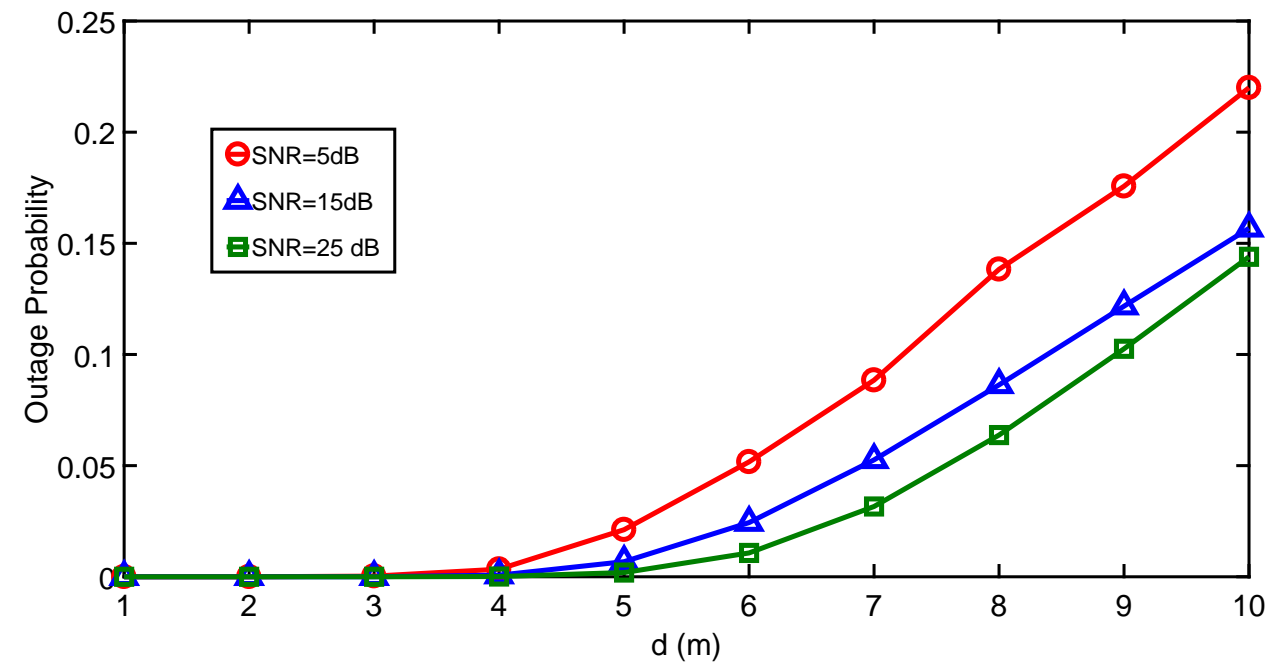

Figure 3. Impact of $d$ on outage probability.

\subsection{Comparison between Paired and Grouped D2D Communications}

The average outage probability achieved by Q-NOMA group D2D communications under different path loss exponents is shown in Figure 4. The performance of paired D2D communication based on OMA is also presented in the figure as a benchmark for comparison. It can be observed that Q-NOMA group D2D achieves overall lower outage probability than the paired D2D communication for different values of path loss. This is because, as opposed to paired D2D, Q-NOMA group D2D communication uses only single transmission, which results in better SINR at the DRs under an interference limited scenario.

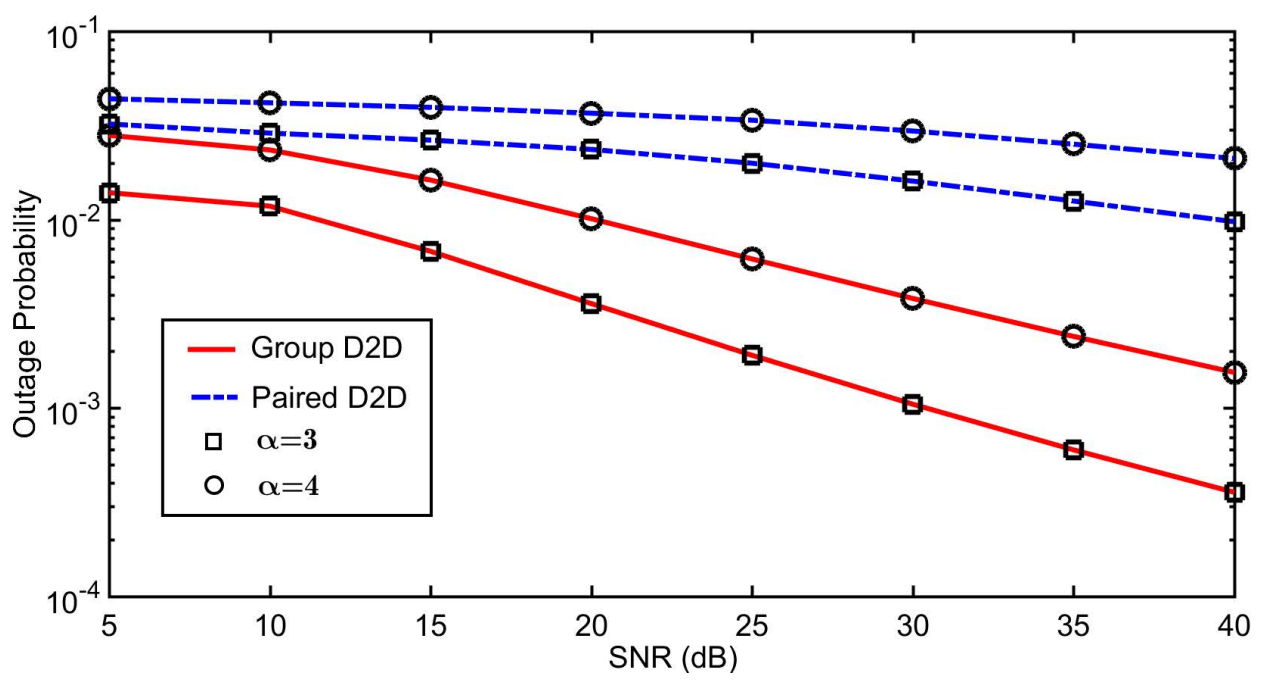

Figure 4. Outage comparison between paired and group D2D. 


\subsection{Comparison between Two Implementations of Q-NOMA Group D2D Communications}

In this section, we compare the performance of two possible implementations of Q-NOMA group D2D communications based on power allocation coefficients Policies I and II. The results for Q-NOMA group D2D communication under Policy I are also obtained by utilising Equation (8), with the exception that $\left\{\varphi_{m}^{\max }\right\}_{m=1}^{M}$ are computed by using fixed power allocation coefficients $\left\{\beta_{m}^{\mathrm{F}}\right\}_{m=1}^{M}$. We consider five different cases for users' targeted rates and compared the outage probabilities achieved by the Q-NOMA group D2D communications under Policies I and II at an SNR of $25 \mathrm{~dB}$. Table 3 summarizes the outage comparison between these two implementations of the group D2D communications. The power allocation coefficients for Policy I are derived as $\beta_{m}^{\mathrm{F}}=\{0.5,0.33,0.17\}_{m=1}^{M}$, whereas those for Policy II are calculated using Equation (2) based on users' targeted rates and are shown in Table 3.

Table 3. Average outage comparison between two implementations of quality of service based non-orthogonal multiple access (Q-NOMA) group device-to-device (D2D) communications.

\begin{tabular}{ccccc}
\hline Case & Targeted Rates, $\left\{\boldsymbol{R}_{\boldsymbol{m}}\right\}_{m=\mathbf{1}}^{\mathbf{3}}$ & Power Allocation Coefficients, $\left\{\boldsymbol{\beta}_{\boldsymbol{m}}\right\}_{m=\mathbf{1}}^{\mathbf{3}}$ & Policy I & Policy II \\
\hline 1. & $\{1,2.5,3\}$ & $\{0.58,0.24,0.18\}$ & 0.04 & 1 \\
2. & $\{1,1.5,2\}$ & $\{0.47,0.3,0.23\}$ & 0.002 & 0.04 \\
3. & $\{0.5,1.5,2\}$ & $\{0.63,0.21,0.16\}$ & 1 & 1 \\
4. & $\{0.9,1,2\}$ & $\{0.42,0.38,0.2\}$ & 1 & 0.001 \\
5. & $\{0.2,2,2.2\}$ & $\{0.85,0.08,0.07\}$ & 0.006 & 0.002 \\
\hline
\end{tabular}

It is observed from the results in Table 3 that Q-NOMA under Policy I achieves lower outage probability in cases 1 and 2, whereas it obtains better outage performance in cases 4 and 5 for Policy II. In addition, the results in case 3 indicate the importance of proper power and rate allocation to avoid situations of complete outage. Moreover, it can be observed that the Q-NOMA group D2D communication under Policy I performs better in cases where the users' targeted rates are significantly apart. This is more suitable to D2D communication scenarios where different users may have diverse QoS requirements.

In order to extend the analysis beyond the case of $M>3$ users, we further present in Table 4 the average outage comparison between paired D2D and Q-NOMA group D2D communications (based on power allocation coefficients Policies I and II). We consider $M=5,7,9$ with $R_{m} \sim \mathcal{U}(0.1,2), \forall m \in M$ and $\rho_{t}=25 \mathrm{~dB}$, where $\mathcal{U}(\cdot, \cdot)$ represents the random uniform distribution function. It can be observed that the results in Table 4 are consistent with those presented in Figure 4, for example, Q-NOMA group D2D communications consistently achieve lower outage probability than paired D2D communications.

Table 4. Average outage comparison between paired device-to-device (D2D) and quality of service based non-orthogonal multiple access (Q-NOMA) group D2D communications $(M>3)$.

\begin{tabular}{cccc}
\hline Total Users, $\boldsymbol{M}$ & Q-NOMA Policy I & Q-NOMA Policy II & Paired D2D \\
\hline 5 & 0.003 & 0.07 & 0.08 \\
7 & 0.007 & 0.06 & 0.12 \\
9 & 0.01 & 0.1 & 0.3 \\
\hline
\end{tabular}

\section{Conclusions}

In this paper, we have proposed Q-NOMA group D2D communications. In order to study the performance of the proposed network, we first derive the Laplace transform of the interference expression, based on which we further derive the closed-form expression for outage probability to analyse the performance of the DRs in the proposed Q-NOMA group D2D communications. The results show that the proposed Q-NOMA group D2D achieves overall lower outage probability than its counterpart paired D2D communication. Furthermore, based on two power allocation coefficient 
policies, we have presented the comparison between two possible implementations of the proposed Q-NOMA group D2D communications. Due to the similar channel conditions and diverse QoS requirements of DRs, the results show that the proposed Q-NOMA implementation based on Policy I is more realistic and suitable than one based on Policy II for group D2D communications. As future work, we plan to investigate and analyse the performance of proposed Q-NOMA group D2D communications in underlay cellular mode, where additional interferences from base stations and cellular users will be considered. Another interesting future direction is to extend the current network model to a framework of MIMO systems. This would require a robust precoder and MIMO-NOMA design to improve the system performance and capacity while mitigating the inter and intra beam interference efficiently.

Acknowledgments: This work has been supported by a PhD Fee and Stipend Scholarship for Asim Anwar from the School of Engineering, Computer and Mathematical Sciences at Auckland University of Technology.

Author Contributions: Asim Anwar and Boon-Chong Seet conceived and designed the study. Asim Anwar derived the Lemmas and Theorems, conducted the simulations and drafted the paper. Boon-Chong Seet and Xue Jun Li critically reviewed the results, proofread and revised the paper.

Conflicts of Interest: The authors declare no conflict of interest.

\section{Appendix A. Proof of Lemma 1}

In the case of GPP, when desired transmitter is at $x_{0} \in \Phi_{G T}$, the $\mathcal{L}_{\mathcal{I}}(s)$ is given by Equation (34) of [21]:

$$
\mathcal{L}_{\mathcal{I}}(s)=\Lambda_{1} \cdot \Lambda_{2}
$$

where

$$
\Lambda_{1}=\exp \left\{2 \pi \lambda_{\mathrm{GT}} \int_{0}^{\infty}\left[\frac{1-a}{1+s r^{-\alpha}}+\frac{a}{1+s r^{-\alpha}} \cdot \int_{0}^{\infty} \int_{0}^{2 \pi} \frac{\tau f_{u}(\tau) d \psi}{1+s\left(r^{2}+\tau^{2}+2 r \tau \cos (\psi)\right)^{-\alpha / 2}} d \tau-1\right] r d r\right\},
$$

and

$$
\Lambda_{2}=\frac{1-a}{1+a}+\frac{2 a}{1+a} \int_{0}^{\infty} \int_{0}^{2 \pi} \frac{\tau f_{u}(\tau) d \psi}{1+s\left(d^{2}+\tau^{2}+2 d \tau \cos (\psi)\right)^{-\alpha / 2}} d \tau
$$

where $1-a$ and $a$ are the probabilities of having one and two transmitters in a group, respectively.

Now let us take $X_{1}$ in Equation (A2) as:

$$
\begin{aligned}
X_{1} & =\int_{0}^{\infty} \int_{0}^{2 \pi} \frac{\tau f_{u}(\tau) d \psi}{1+s\left(r^{2}+\tau^{2}+2 r \tau \cos (\psi)\right)^{-\alpha / 2}} d \tau \\
& =\frac{2}{R_{D}^{2}} \int_{0}^{R_{D}} \underbrace{\int_{0}^{2 \pi} \frac{d \psi}{1+s\left(r^{2}+\tau^{2}+2 r \tau \cos (\psi)\right)^{-\alpha / 2}}}_{X_{2}} d \tau
\end{aligned}
$$

where $f_{u}(\tau)=\frac{2 \tau}{R_{D}^{2}}$ if $0 \leq \tau \leq R_{D}$.

It is challenging to solve integral $X_{2}$ in Equation (A4). As such, we approximate it by applying the Gaussian-Chebyshev quadrature as [30]:

$$
X_{2} \approx \sum_{n=1}^{N} \frac{\varphi_{n}}{1+s\left(r^{2}+\tau^{2}+2 r \tau \cos \left(\pi t_{n}\right)\right)^{-\alpha / 2}},
$$

where $\varphi_{n}=\pi \omega_{n} \sqrt{1-\theta_{n}^{2}}, \omega_{n}=\frac{\pi}{N}, \theta_{n}=\cos \left(\frac{2 n-1}{2 N} \pi\right), t_{n}=1+\theta_{n}$ and $N$ is the complexity-accuracy tradeoff parameter. 
Based on Equation (A5), $X_{1}$ can now be expressed as:

$$
X_{1}=\frac{2}{R^{2}} \int_{0}^{R_{D}} \sum_{n=1}^{N} \frac{\varphi_{n} \tau^{2}}{1+s\left(r^{2}+\tau^{2}+2 r \tau \cos \left(\pi t_{n}\right)\right)^{-\alpha / 2}} d \tau
$$

Note that it is challenging to solve Equation (A6) analytically. In order to obtain insightful results, we approximate it by applying Gaussian-Chebyshev quadrature as:

$$
X_{1}(r) \approx \sum_{v=1}^{V} \sum_{n=1}^{N} \frac{2 R_{D} \varphi_{n} \omega_{v} \sqrt{1-\vartheta_{v}^{2}} k_{v}^{2}}{1+s\left(r^{2}+R_{D}^{2} k_{v}^{2}+2 r R_{D} k_{v}^{2} \cos \left(\pi t_{n}\right)\right)^{-\alpha / 2}}
$$

where $\omega_{v}=\frac{\pi}{V}, \vartheta_{v}=\cos \left(\frac{2 v-1}{2 V} \pi\right), k_{v}=\frac{1}{2}\left(\vartheta_{v}+1\right)$ and $V$ is the complexity-accuracy tradeoff parameter.

Based on Equation (A7), $\Lambda_{1}$ in Equation (A2) is re-written as:

$$
\begin{aligned}
\Lambda_{1} & =\exp \left\{2 \pi \lambda_{\mathrm{GT}} \int_{0}^{\infty}\left[\frac{1-a}{1+s r^{-\alpha}}+\frac{a X_{1}(r)}{1+s r^{-\alpha}}-1\right] r d r\right\} \\
& =\exp \left\{-2 \pi \lambda_{\mathrm{GT}} \int_{0}^{\infty} \frac{a\left(1-X_{1}(r)\right)+s r^{-\alpha}}{1+s r^{-\alpha}}\right\} .
\end{aligned}
$$

Next, we apply Gauss-Laguerre quadrature to approximate the integral in Equation (A8). Hence, $\Lambda_{1}$ can be expressed after approximation as:

$$
\Lambda_{1}=e^{-2 \pi \lambda_{\mathrm{GT}} \sum_{p=1}^{P} \Omega_{p} \frac{a\left(1-X_{1}\left(r_{p}\right)\right)+s r_{p}^{-\alpha}}{1+s r_{p}^{-\alpha}}} .
$$

Following the same approximation procedure for $X_{1}$ and applying Gaussian-Chebyshev quadrature twice, $\Lambda_{2}$ in Equation (A3) is given as:

$$
\Lambda_{2}(d)=\frac{1-a}{1+a}+\frac{2 a}{1+a} \sum_{j=1}^{S} \sum_{i=1}^{Q} \frac{\varrho_{i} \xi_{j}}{1+s\left(d^{2}+R_{D}^{2} z_{j}^{2}+2 d R_{D} z_{j} \cos \left(\pi x_{i}\right)\right)^{-\alpha / 2}}
$$

where $\varrho_{i}=\pi \omega_{i} \sqrt{1-\eta_{i}^{2}}, \omega_{i}=\frac{\pi}{Q}, \eta_{i}=\cos \left(\frac{2 i-1}{2 Q} \pi\right), x_{i}=\eta_{i}+1, \xi_{j}=2 R_{D} \omega_{j} \sqrt{1-\Theta_{j}^{2}} z_{j}^{2}$, $\Theta_{j}=\cos \left(\frac{2 j-1}{2 S} \pi\right), z_{j}=\frac{1}{2}\left(\Theta_{j}+1\right), \omega_{j}=\frac{\pi}{S}$ and $Q, S$ are the complexity-accuracy trade-off parameters.

Finally, the result in Lemma1 is obtained by multiplying Equations (A9) and (A10).

\section{Appendix B. Proof of Theorem 1}

In order to obtain $\mathbb{P}_{m}$, we require $F_{h}$. Since, all wireless links exhibit Rayleigh fading and the DRs are uniformly distributed inside disc $D$ centered at the location of test GT, the CDF $F_{h}$ can be expressed as [31]:

$$
F_{h}(y)=\frac{2}{R_{D}^{2}} \int_{0}^{R_{D}}\left(1-e^{-z^{\alpha} y}\right) z d z
$$

It is challenging to solve the above integral. As such, we approximate it by applying Gaussian-Chebyshev quadrature as:

$$
F_{h}(y)=\sum_{l=1}^{L} b_{l} e^{-c_{l} y}
$$


Based on Equation (A12), $\mathbb{P}_{m}$ in Equation (5) can be expressed as:

$$
\begin{aligned}
\mathbb{P}_{m} & =\int_{0}^{\infty} F_{h}\left(\frac{\varphi_{m}^{\max }(\rho x+1)}{\rho_{t}}\right) f_{I}(x) d x \\
& =\sum_{l=1}^{L} \int_{0}^{\infty} e^{-\frac{c_{l} \varphi_{m}^{\max }(\rho x+1)}{\rho_{t}}} f_{I}(x) d x \\
& =\sum_{l=1}^{L} b_{l} e^{-\frac{c_{l} \varphi_{m}^{\max }}{\rho_{t}}} \int_{0}^{\infty} e^{-\frac{c_{l} \varphi_{m x}^{\max } \rho x}{\rho_{t}}} d x \\
& =\sum_{l=1}^{L} b_{l} e^{-\frac{c_{l} \varphi_{m}^{\max }}{\rho_{t}}} \mathcal{L}_{I}\left(\frac{c_{l} \varphi_{m}^{\max } \rho}{\rho_{t}}\right),
\end{aligned}
$$

where $f_{I}(x)$ is the PDF of interference $I$ and the last step follows from the definition of Laplace transform. This proves the result in Theorem 1 .

\section{References}

1. Akyildiz, I.F.; Nie, S.; Lin, S.C.; Chandrasekaran, M. 5G roadmap: 10 key enabling technologies. Comput. Netw. 2016, 106, 17-48.

2. Wong, V.W.S.; Schober, R.; Ng, D.W.K.; Wang, L.C. Key Technologies for 5 G Wireless Systems; Cambridge University Press: Cambridge, UK, 2017.

3. Timotheou, S.; Krikidis, I. Fairness for Non-Orthogonal Multiple Access in 5G Systems. IEEE Signal Process. Lett. 2015, 22, 1647-1651.

4. Anwar, A.; Seet, B.C.; Li, X.J. PIC-based receiver structure for 5G downlink NOMA. In Proceedings of the 10th International Conference on Information, Communications and Signal Processing (ICICS), Singapore, 2-4 December 2015.

5. Ding, Z.; Liu, Y.; Choi, J.; Sun, Q.; Elkashlan, M.; Chih-Lin, I.; Poor, H.V. Application of Non-Orthogonal Multiple Access in LTE and 5G Networks. IEEE Commun. Mag. 2017, 55, 185-191.

6. Wei, Z.; Yuan, J.; Ng, D.W.K.; Elkashlan, M.; Ding, Z. A Survey of Downlink Non-orthogonal Multiple Access for 5G Wireless Communication Networks. arXiv 2016, arXiv:abs/1609.01856.

7. Saito, Y.; Benjebbour, A.; Kishiyama, Y.; Nakamura, T. System-level performance evaluation of downlink non-orthogonal multiple access (NOMA). In Proceedings of the IEEE 24th Annual International Symposium on Personal, Indoor, and Mobile Radio Communications (PIMRC), London, UK, 8-11 September 2013.

8. Lv, L.; Chen, J.; Ni, Q.; Ding, Z. Design of Cooperative Non-Orthogonal Multicast Cognitive Multiple Access for 5G Systems: User Scheduling and Performance Analysis. IEEE Trans. Commun. 2017, 65, 2641-2656.

9. Kimy, B.; Lim, S.; Kim, H.; Suh, S.; Kwun, J.; Choi, S.; Lee, C.; Lee, S.; Hong, D. Non-orthogonal Multiple Access in a Downlink Multiuser Beamforming System. In Proceedings of the IEEE Military Communications Conference (MILCOM), San Diego, CA, USA, 18-20 November 2013.

10. Andrews, J.G.; Buzzi, S.; Choi, W.; Hanly, S.V.; Lozano, A.; Soong, A.C.K.; Zhang, J.C. What Will 5G Be? IEEE J. Sel. Areas Commun. 2014, 32, 1065-1082.

11. Fodor, G.; Dahlman, E.; Mildh, G.; Parkvall, S.; Reider, N.; Miklós, G.; Turányi, Z. Design aspects of network assisted device-to-device communications. IEEE Commun. Mag. 2012, 50, 170-177.

12. Doppler, K.; Rinne, M.; Wijting, C.; Ribeiro, C.B.; Hugl, K. Device-to-device communication as an underlay to LTE-advanced networks. IEEE Commun. Mag. 2009, 47, 42-49.

13. Lin, X.; Andrews, J.G.; Ghosh, A. Spectrum Sharing for Device-to-Device Communication in Cellular Networks. IEEE Trans. Wirel. Commun. 2014, 13, 6727-6740.

14. Zhang, Z.; Ma, Z.; Xiao, M.; Ding, Z.; Fan, P. Full-Duplex Device-to-Device-Aided Cooperative Nonorthogonal Multiple Access. IEEE Trans. Veh. Technol. 2017, 66, 4467-4471.

15. Ali, K.S.; ElSawy, H.; Alouini, M.S. Modeling Cellular Networks With Full-Duplex D2D Communication: A Stochastic Geometry Approach. IEEE Trans. Commun. 2016, 64, 4409-4424.

16. Afshang, M.; Dhillon, H.S. Spatial modeling of device-to-device networks: Poisson cluster process meets Poisson Hole Process. In Proceedings of the 49th Asilomar Conference on Signals, Systems and Computers, Pacific Grove, CA, USA, 8-11 November 2015. 
17. Palombara, C.L.; Tralli, V.; Masini, B.M.; Conti, A. Relay-Assisted Diversity Communications. IEEE Trans. Veh. Technol. 2013, 62, 415-421.

18. Sun, H.; Xu, Y.; Hu, R.Q. A NOMA and MU-MIMO Supported Cellular Network with Underlaid D2D Communications. In Proceedings of the IEEE 83rd Vehicular Technology Conference (VTC Spring), Nanjing, China, 15-18 May 2016.

19. Zhao, J.; Liu, Y.; Chai, K.K.; Chen, Y.; Elkashlan, M.; Alonso-Zarate, J. NOMA-Based D2D Communications: Towards 5G. In Proceedings of the 2016 IEEE Global Communications Conference (GLOBECOM), Washington, DC, USA, 4-8 December 2016.

20. Shi, Z.; Ma, S.; ElSawy, H.; Yang, G.; Alouini, M. Cooperative HARQ Assisted NOMA Scheme in Large-scale D2D Networks. arXiv 2017, arXiv:abs/1707.03945.

21. Guo, A.; Zhong, Y.; Zhang, W.; Haenggi, M. The Gauss Poisson Process for Wireless Networks and the Benefits of Cooperation. IEEE Trans. Commun. 2016, 64, 1916-1929.

22. Yang, Z.; Ding, Z.; Wu, Y.; Fan, P. Novel Relay Selection Strategies for Cooperative NOMA. IEEE Trans. Veh. Technol. 2017, doi:10.1109/TVT.2017.2752264.

23. Ding, Z.; Dai, H.; Poor, H.V. Relay Selection for Cooperative NOMA. IEEE Wirel. Commun. Lett. 2016, 5, 416-419.

24. Ding, Z.; Dai, L.; Poor, H.V. MIMO-NOMA Design for Small Packet Transmission in the Internet of Things. IEEE Access 2016, 4, 1393-1405.

25. Sun, Y.; Ng, D.W.K.; Zhu, J.; Schober, R. Multi-Objective Optimization for Robust Power Efficient and Secure Full-Duplex Wireless Communication Systems. IEEE Trans. Wirel. Commun. 2016, 15, 5511-5526.

26. Zabini, F.; Bazzi, A.; Masini, B.M.; Verdone, R. Optimal Performance Versus Fairness Tradeoff for Resource Allocation in Wireless Systems. IEEE Trans. Wirel. Commun. 2017, 16, 2587-2600.

27. Ding, Z.; Yang, Z.; Fan, P.; Poor, H.V. On the Performance of Non-Orthogonal Multiple Access in 5G Systems with Randomly Deployed Users. IEEE Signal Process. Lett. 2014, 21, 1501-1505.

28. Liu, Y.; Ding, Z.; Elkashlan, M.; Yuan, J. Nonorthogonal Multiple Access in Large-Scale Underlay Cognitive Radio Networks. IEEE Trans. Veh. Technol. 2016, 65, 10152-10157.

29. Men, J.; Ge, J.; Zhang, C. Performance Analysis of Nonorthogonal Multiple Access for Relaying Networks Over Nakagami-m Fading Channels. IEEE Trans. Veh. Technol. 2017, 66, 1200-1208.

30. Hiderband, E. Introduction to Numerical Analysis; Dover: New York, NY, USA, 1987.

31. Ding, Z.; Poor, H.V. Cooperative Energy Harvesting Networks With Spatially Random Users. IEEE Signal Process. Lett. 2013, 20, 1211-1214.

(C) 2017 by the authors. Licensee MDPI, Basel, Switzerland. This article is an open access article distributed under the terms and conditions of the Creative Commons Attribution (CC BY) license (http://creativecommons.org/licenses/by/4.0/). 